REVIEW

\title{
Optimising neonatal transfer
}

A C Fenton, A Leslie, C H Skeoch

Arch Dis Child Fetal Neonatal Ed 2004;89:F215-F219. doi: 10.1136/adc.2003.019711

Services for neonatal intensive care in the United Kingdom have evolved in a largely unplanned fashion. Units of different sizes provide various amounts of intensive care, and, with a few exceptions, there is little or no formal regional or subregional organisation. Chronic underresourcing and the salvaging of ever more complex infants have resulted in tertiary neonatal intensive care units operating at full capacity most of the time, a situation compounded by a chronic national shortage of nursing staff. These factors have in turn resulted in an increase in requirements for emergency perinatal transfers.

See end of article for authors' affiliations

...........................

Correspondence to: Dr Fenton, Newcastle Neonatal Service, Ward 35, Royal Victoria Infirmary, Queen Victoria Road, Newcastle upon Tyne NEl 4LP, UK; a.c.fenton@ncl.ac.uk

Accepted 25 April 2003
$\mathrm{R}$ ecent studies have suggested that "inappropriate" antenatal transfers (because of lack of intensive care cots) are commonplace, ${ }^{1}$ although not all such infants either deliver immediately or subsequently require intensive care. ${ }^{2}$ Where antenatal transfer is not possible, postnatal transfer is necessary for sick infants born in hospitals without appropriate facilities for continuing intensive care. These transfers are often performed by staff with little experience of or guidance in working in the transfer environment, using unfamiliar equipment. The proposed recommendations for the reconfiguration of neonatal services ${ }^{3}$ will almost certainly increase the need for postnatal transfers. This will include transfer into designated centres for intensive care or specialist services and return transfers for continuing care once intensive support is no longer needed.

Regionalisation of neonatal intensive care presents a major opportunity to establish safe, efficient transfer networks. For this strategy to be successful and adverse incidents to be minimised, a review of existing neonatal transfer arrangements in many regions will be required. Neonatal transfer should be seen as an integral part of the continuum of care that a particular infant may require and this review examines the early stages of that process, with particular emphasis on acute postnatal transfer. Most of the following principles, however, also apply to non-acute transfers, and timely availability of safe backtransfer is another key component of an effective neonatal intensive care service. With intensive care cots often scarce at tertiary centres, it is essential that infants who are fit to return to a local centre are transferred promptly

The proposed managed clinical network model for neonatal intensive care services assumes that transfer services will be provided centrally for the network. This contrasts substantially with the present situation in many areas of the United Kingdom, where the unreliable availability of many tertiary transfer teams has ensured that even the smallest neonatal units generally possess some interhospital transfer equipment, which is used rarely. For a transfer team to be reliably available, the nursing and medical staff involved should be supernumerary to the staffing requirements of the base unit and/or work as a "stand alone" service, independent of a hospital. Factors influencing the use of particular models for providing neonatal transfer within Europe have been examined, ${ }^{4}$ and it is clear that there is not a "one size fits all" solution. Within each network, the transfer service should ideally be tailor made to suit the region's demography, geography, and specific requirements. For this to be achieved, there are several steps that should be undertaken. It is highly desirable that a multidisciplinary group representing all parties involved is established early on in the process. This should include clinicians, nurses, midwives, managers, commissioners, the ambulance services involved, and health and safety representatives.

\section{WORKLOAD}

Planning for a centralised transport service has several appreciable tensions. If the area or population being covered is too large, transfers may be delayed for several hours until the team becomes available. Conversely, the workload for a team covering too small a population will render it cost ineffective. Data from European regions where transfer services have been centralised suggest that a population base of 4-5 million is large enough to support a dedicated service performing both acute and return transfers without the workload being overwhelming. ${ }^{5}$ Cooperation between smaller clinical networks may be necessary to share the additional costs of a supernumerary transfer service.

The workload of a neonatal transfer service is likely to be highly variable. To achieve an overview of the likely transfer activity in a defined region, certain information is required, including current numbers of transfers, severity of illness, and timing and geography of transfers. These details are best collected prospectively unless existing systems record all activity, including return transfers.

For example, in the Trent Region in 1997 the population was approximately 4.7 million with 63000 births annually. Examination of the transfer workload over a three month period showed that there was a median of one acute

Abbreviations: CPAP, continuous positive airways pressure; iNO, inhaled nitric oxide 
transfer a day, but that the range was from zero to five. ${ }^{6}$ Similarly, in the preparation of the Acute Services Review in Scotland, a three month daily telephone enquiry to each hospital in the country involved in the transfer of infants provided invaluable data leading to a national service. This showed that there were about 15 transfers per thousand births per annum, a level very similar to that in Trent. ${ }^{7}$ Modelling exercises based on knowledge of real transfer patterns may help to define the appropriate geographical area or population to be covered by a transfer service, and several surveys have been undertaken recently that should yield valuable data for planning purposes. The establishment of dedicated transfer services that cover substantial populations will undoubtedly reduce service pressures on the medical and nursing staff at many hospitals and create collaborative opportunities for interested personnel from several referral units. Several areas of the UK, including London/South-East England, Yorkshire, and Scotland, have made appreciable recent progress in improving transport organisation and availability. The key to these reorganisations has been a willingness of commissioning bodies and healthcare professionals to work beyond both traditional and new geographical boundaries.

\section{REFERRAL PROCESS}

The initial care of a sick infant is a key factor in its long term outcome. It therefore follows that the transfer process really begins with the recognition by referring hospital staff that a particular infant is unwell and may require treatment that is unavailable at that hospital. It is, however, essential that the referring hospital is able to provide an appropriate standard of care from birth up to the point of transfer. This has been addressed in part by having national guidelines and training for neonatal resuscitation. ${ }^{8}$ Subsequent care should be directed towards stabilising the infant until the arrival of the transfer team. The transfer then becomes simply a part of the continuing care that the infant requires, obviating the need for a "flying squad" resuscitation service. The latter puts staff at risk from injury during high speed journeys and in most cases will not affect outcome.

Once an infant is identified as potentially needing transfer, it is essential that referring clinicians spend as little time as possible initiating that process and as much time as necessary caring for the infant. Clear referral pathways and protocols should be established, ideally requiring a single call to the transfer service, which then takes on the responsibility of moving the infant to an appropriate cot. A simple solution is to establish a dedicated telephone "hotline" within a region through which the transfer service may be immediately accessed 24 hours a day. From this point, the transfer service assumes some responsibility towards the patient, shared with the referring clinician.

For this approach to be successful, calls to a "hotline" must have ready access to personnel with appropriate expertise. Requests for transfer need to be triaged according to how unwell the patient is, advice given to the referring clinician pending the arrival of the transfer team, and the transfer team mobilised. Within a particular network, there may be local factors relating to individual units that will influence the response that occurs. In general terms, however, the population distribution across the United Kingdom and the relatively short distances between hospitals in most regions means that, with the potential exception of the more remote units in Scotland, transfer response times should not be a major issue.

Clearly the accepting clinician will need the most up to date information available on the infant. An understanding of the natural history of the presenting problem is important in addition to the effect that a transfer may have on the patient's condition. The infant may benefit from treatments unavailable in the referring hospital, for example inhaled nitric oxide, and the team will need to take this "nonstandard" equipment with them. Finally the person dealing with the referral needs to understand the logistics involved in the transfer process, particularly how long the transfer is likely to take and what type of vehicle is to be used. It therefore follows that dealing with requests for transfer should not be delegated to the most junior medical or nursing staff; whether requests are routed directly to a consultant neonatologist, or to a member of the transfer team will depend on local organisational factors and expertise. All details of the referral, advice given, and action taken should be recorded contemporaneously to ensure clinical continuity and to allow audit.

There may be concerns that the establishment of managed clinical networks will result in a deskilling of clinicians in referring units. Although they may have less experience with long term neonatal intensive care, it is likely that they will continue to be exposed to similar volumes of patients requiring resuscitation and stabilisation, which will maintain skills. This has clearly been the experience in the former Northern region.

\section{AMBULANCE SERVICE INTERFACE}

Ambulance services are a key element of transfers. Simplistically, all that is required is the ability to put a transport incubator system in an ambulance with appropriate staff and ancillary equipment and convey them to and from the referring unit. Within this seemingly straightforward clinical scenario lies a catalogue of potential organisational pitfalls and a myriad of equipment and Health and Safety regulations. The latter were often ignored in the past. European Committee for Standardization (CEN) regulations (CEN 13976-2) will soon dictate maximum weights of equipment, standards for locking incubator trolleys in road vehicles, and many other aspects of transfer equipment systems. Any individual or organisation failing to follow these regulations will be culpable in the event of an accident, with implications for insurance cover for participating staff. Clinical governance is therefore a major issue. Close cooperation with the ambulance service and equipment engineers can result in good quality fixation systems which comply with CEN regulations. This should reduce the potential for injury to patients, transfer team, and ambulance staff.

As with clinical situations, working with ambulance services requires clear communication protocols written in a language that all parties can understand. This avoids ambiguity about response times and vehicle requirements. Regular service based discussions enable problem areas to be identified and solutions developed. This type of forum will also alert clinicians to proposed changes to ambulance fleets, which in turn may require planned replacement of incubator trolleys.

\section{TRAINING}

A number of studies ${ }^{10-12}$ have shown that it is possible to improve the condition of infants on completion of transfer by deploying trained teams and that these infants have lower rates of in transit deterioration. Studies of long term outcomes in infants after transfer are confounded by the long periods of complex intensive care many of them require. ${ }^{13-15}$ The future organisation of neonatal care provides an opportunity for ensuring that no patients are transferred by inexperienced, untrained personnel.

Training in the care of infants needing transfer should be undertaken by both personnel undertaking transfers and all staff who may have to care for infants before transfer. There are at present no agreed national standards or competencies 
for training in transfer, although this may well be an area, like resuscitation at birth, where a life support course model is applicable. Such a course (PNeoSTaR) has been developed with the help of the Advanced Life Support Group, and may help raise standards nationally. Agreed common local policies and practices may smooth the arrival and departure of the team, and a broad approach to stabilising babies for transfer is outlined below.

\section{STAFFING}

All staff attending transfers should have transfer training and experience. All transfers need a nurse, and a second team member is needed on acute transfers, where the infant is or may become seriously unwell. This is usually a doctor at specialist registrar level or above, but recent UK data suggest that it is practical and safe to use advanced neonatal nurse practitioners in this role. ${ }^{16}{ }^{17}$ Reductions in numbers of and hours worked by paediatricians in training is likely to limit their role in transfer services in the future. Ambulance personnel have historically played a minimal role in the provision of patient care during neonatal transfer in the United Kingdom, although in other countries (notably North America) paramedics play a major role in neonatal transfer teams. This is an area that may warrant development.

\section{CLINICAL ISSUES}

\section{Stabilisation before transfer}

This period comprises two phases: (a) from when a decision to transfer is made until the transfer team arrives, during which care is delivered by the local staff; $(b)$ after the team's arrival. The aim in both of these phases is to resuscitate and stabilise the infant in preparation for transfer. The essential elements of this process are common to the referring and transfer teams, and are summarised in table l. The extent to which these issues are attended to before the transfer team arrives will depend on the time and human resources available at the referring unit. The interface between transfer team and referring unit is not always a smooth one, ${ }^{18}$ and an appreciation of each others' perspectives and priorities is essential. Once again common treatment protocols and two way communication between referring and transfer teams will minimise these potential problems. This may be formalised in the setting of a managed clinical network and should offer no surprises.

\section{Safety}

Staff are central to any system and their safety and welfare is of paramount importance. The transfer environment is hostile and staff should be trained in working under transfer conditions. Loading and unloading equipment is potentially hazardous, and, once loaded, all equipment must be adequately secured. Protective clothing should be made available for staff, both to allow them to be seen at night and to protect them from temperature extremes.

Considerable improvements have been made in recent years in the availability of equipment for transfer that is crash stable. Several fixation systems for trolleys have been crash tested to the proposed European standard, ${ }^{22}$ and neonatal transfer teams should be converting to these devices if they have not already done so.

Fixation devices are challenged in road traffic accidents by a combination of heavy equipment and speed of impact. For these reasons, neonatal transfer teams should take two further steps beyond using crash stable fixation, to fully promote safety:

- Limit the weight of equipment. Maximum transfer system weight should be $140 \mathrm{~kg}$, excluding the trolley, in line with European standards (CEN 13976-2).
- Restrict the use of emergency driving to situations where there is likely to be appreciable clinical benefit from decreased journey time. In the United States, ambulances were found to be more likely to be involved in road traffic accidents and to result in higher injury rates when lights and sirens were being used. ${ }^{23}$

Staff undertaking transfers by air require additional training specific to the aircraft they are using with regard to both safety in flight and the equipment carried by the aircraft.

\section{Equipment}

It may be important to standardise transport equipment within a managed clinical network. Achieving this will need multiagency discussion and working groups with wide representation (including ambulance services). Issues of data collection and analysis, option appraisal, and costings should be considered. Comprehensive guidance on equipment for transport $^{24}$ and a review of current systems in use in Europe ${ }^{25}$ have been published elsewhere. Several issues are worthy of some further discussion.

\section{Inhaled nitric oxide (iNO)}

Tertiary transfer teams should have the facility to administer iNO to infants with persistent pulmonary hypertension during transfer. ${ }^{26}$ As there is at present no commercially available portable iNO delivery system, each centre needs to make provision for this. There are substantial health and safety issues to be attended to for iNO to be safely available for transport. A portable system may be best configured as an optional "bolt on" facility that may be mounted as an extra on the incubator trolley when required. Alternatively, an integral system may be used, the iNO cylinder being removed when not in use. Both of these options avoid carrying a hazardous gas on most neonatal transfers. The iNO system and its components must be secure in transit, and operational protocols and training should ensure that robust procedures are in place to deal with gas leaks. A simple system comprising a small cylinder of NO, a flowmeter, and an $\mathrm{NO} / \mathrm{NO}_{2}$ monitor with good battery life is sufficient for most journeys. Scavengers may be placed in the expiratory line of the ventilator (although this may be unnecessary if adequate air handling systems are operating in the vehicle). ${ }^{27}$ Consideration should also be given to environmental monitoring. This is less of a concern since the introduction of cylinders with a reduced concentration of iNO. The smallest available iNOmax cylinder contains 307 litres with a concentration of NO of $400 \mathrm{ppm}$, which if accidentally discharged while full would result in an environmental NO level of $14 \mathrm{ppm}$ in a volume of $9 \mathrm{~m}^{3}$, assuming complete distribution and no attempts to ventilate the area. This volume is similar to that within a closed vehicle, and this NO level is significantly below mandated safety standards. There is understandable concern from the aviation operators about the safety of iNO in pressurised aircraft. It is a legal requirement that a dangerous goods assessment is carried out and highly desirable that protocols are in place before the use of iNO in this situation. Such a protocol has been jointly developed in Scotland by clinicians, the Scottish Ambulance Service, and Gama Aviation.

\section{High frequency oscillatory ventilation}

Infants who need such ventilation in transit currently present a problem, as there is no oscillatory ventilation option on commonly available transport ventilators. Clinical teams will usually have to convert oscillation to standard ventilation for transfer, and there is anecdotal experience of using iNO to support this conversion and make transfer possible in very unstable infants. 
Table 1 Outline of key issues in stabilising neonates for transfer ${ }^{19}$

\section{Airway/breathing}

- Should the baby be intubated before transfer? A lower threshold for intubation should be used than on the neonatal intensive care unit, to minimise the need to intervene in transit. In an infant $>30$ weeks gestation, if the vital signs (pulse, blood pressure, respiratory rate, temperature) have been consistently stable in oxygen $<50 \%$ and if the $\mathrm{PaCO}_{2}$ is normal, it may be acceptable to move the baby without intubation. If the infant is: unstable

has a rising oxygen requirement $>50 \%$

has a rising $\mathrm{PaCO}_{2}$

has recurrent apnoed is $<30$ weeks gestation

then intubation and respiratory support is highly likely to be required, at least for the duration of the journey.

- If already intubated, the endotracheal tube (ETT) must be correctly positioned and secure. ETTs must be secured to a high standard, to avoid accidental extubation in transit.

- Adequate respiratory support must be given.

- Surfactant must be administered if indicated.

Circulation

- Arterial access, if not already established, should be considered in infants who require repeated blood gas analysis or accurate blood pressure measurement. If siting a line will not influence practice before or during the journey, then it may be acceptable to delay this until after the transfer.

- Correct positioning and security of the catheter must be checked.

- Circulation with fluids and/or inotropes should be supported early, as indicated.

Temperature

- Assess temperature and consider the support required for transfer.

- Use temperature maintenance adjuncts, such as chemical gel mattresses. ${ }^{20} 21$

Blood glucose

- Measure and stabilise blood glucose.

- Secure intravenous access.

Infection

- Screen for infection as indicated.

- Start treatment.

Parents' information and wishes

- Discuss plans with parents. Ascertain their plans about travelling to referral unit. Liaise with midwifery staff about maternal transfer.

Information

- Ensure the team at the referral unit will have all the necessary information to advance the care of the baby.

\section{Continuous positive airways pressure (CPAP)}

CPAP is used predominantly as a weaning tool, but a number of units are investigating its use during the acute stages of respiratory distress syndrome, following a Scandinavian model which includes surfactant administration and developmentally supportive care. A battery driven version of the EME infant flow CPAP system is available (EME, Brighton, Sussex, UK), but its use during acute transfer has not been reported. Even with trained and experienced staff, the transport environment remains a potentially hazardous one, and further studies are needed to establish which, if any, patients with respiratory distress syndrome who would normally be intubated may be safely transferred on CPAP. Intubation remains the optimum method of airway protection and respiratory support for transfer.

\section{Blood gas measurement in transit}

Transcutaneous estimation of blood gases is widely used in neonatal intensive care units. The technique may also be applied in transport, provided that the monitors are small and have a good external battery facility. A randomised controlled study ${ }^{28}$ found that preterm infants transferred with transcutaneous carbon dioxide monitoring completed the transfer with considerably better blood gases and were also able to have their ventilation weaned during transfer, compared with infants transferred without the monitoring. Portable blood gas analysers are available, but there are relatively few data on their use during transfer.

\section{AUDIT}

No service would be complete without a method of analysing its efficiency. For transfers, this requires prospective data collection from the time of initial referral until the team return to their base unit and should include technical, logistical, and clinical data. Web based systems allow the viewing of up to date data by relevant professional groups and can also be used to ensure that all participating staff are made aware of critical incidents or equipment warnings, such as those from the Medical Devices Agency.

\section{CONCLUSION}

It is six years since Field et al ${ }^{29}$ called for a reorganisation of neonatal transfer services in the United Kingdom. Relatively little progress has been made since then in terms of regional transfer service development. The establishment of managed clinical networks will require changes in all aspects of how perinatal care is delivered. Transfer is a key component of an integrated perinatal service. Acutely sick infants require transfer for specialist care, and timely return helps to keep tertiary beds occupied by appropriate patients. Provision of an appropriately funded, organised transfer service is central to the operation of a managed clinical network for neonatal intensive care.

\section{Authors' affiliations}

A C Fenton, Newcastle Neonatal Service, Ward 35, Royal Victoria Infirmary, Queen Victoria Road, Newcastle upon Tyne NE1 4LP, UK A Leslie, Nottingham Neonatal Service, Neonatal Intensive Care Unit, City Hospital, Hucknall Road, Nottingham NG5 1PB, UK

C H Skeoch, Neonatal Unit, Princess Royal Maternity, Glasgow G32

2ER, Scotland, UK

\section{REFERENCES}

1 Parmanum J, Field D, Rennie J, et al. National census of availability of neonatal intensive care. British Association for Perinatal Medicine. BMJ 2000:321:727-9.

2 Fenton AC, Ainsworth SB, Sturgiss SN. Population-based outcomes after antenatal transfer. Paediatr Perinat Epidemiol 2002;16:278-85.

3 Department of Health. Neonatal intensive care review: strategy for improvement. Report of the Neonatal Intensive Care Services Review Group. London: DOH, 2003. 
4 Agostino R, Fenton AC, Kollee LAA, et al. Organisation of neonatal transport in Europe. Prenat Neonatal Med 1999;4(suppl 1):20-34.

5 Roy RND, Langford S, Chabernaud JL, et al. Newborn transport around the world. Semin Neonatol 1999:4:219-35.

6 Leslie A, Bohin S, Gibson A, et al. Transport workload in Trent Region: issues in planning centralised neonatal transport services. Neonatal Nurses Association yearbook. London: Neonatal Nurses Association, 1999.

7 Jackson L, Deans S, Skeoch CH. Babies on the move. Incorporated in report to CMO (Scotland). Working Group on the Transport of Critically III and Injured Children 2002. Submitted to Scottish Executive.

8 Resuscitation Council (UK). Resuscitation guidelines. London: Resuscitation Council, 2000:35-51.

9 Resuscitation Council (UK). Newborn life support course provider manual. London: Resuscitation Council, 2001.

10 Chance GW, Matthew JD, Gash J, et al. Neonatal transport: a controlled study of skilled assistance. J Pediatr 1978;93:662-6.

11 Leslie A, Stephenson T. Audit of neonatal intensive care transport: closing the loop. Acta Paediatr 1997;86:1253-6.

12 Hood JL, Cross A, Hulka B, et al. Effectiveness of the neonatal transport team. Crit Care Med 1983;11:419-23.

13 Bowman E, Doyle LW, Murton L, et al. Increased mortality of preterm infants transferred between tertiary perinatal centres. BMJ 1988;297:1098-100.

14 Harding JE, Morton SM. Adverse effects of neonatal transport between level iii centres. J Pediatr Child Health 1993;29:146-9.

15 Harding JE, Morton SM. Outcome of neonates transported between level iii centres depends upon centre of care. J Pediatr Child Health 1994;30:389-92.

16 Leslie A, Stephenson T. Neonatal transfers by advanced neonatal nurse practitioners and paediatric registrars. Arch Dis Child Fetal Neonatal Ed 2003;88:F509-12.
17 Leslie A, Bose C. Nurse-led neonatal transport. Semin Neonatol 1999:4:265-71.

18 Leslie A, Middleton D. Give and take in neonatal transport: communication hazards in handover. J Neonatal Nurs 1995;1:27-31.

19 Neonatal resuscitation and stabilisation. In: Barry P, Leslie A, eds. Paediatric and neonatal critical care transport. London: BMJ Books, 2003.

20 L'Herault J, Petroff L, Jeffrey J. The effectiveness of a thermal mattress in stabilising and maintaining boby temperature during the transport of very low birth weight newborns. Appl Nurs Res 2001;14:210-19.

21 Nielson $\mathrm{H}$. Jung A, Atherton S. Evaluation of the Porta-Warm mattress as a source of heat for neonatal transport. Pediatrics 1976;58:500-4.

22 McKay S, Cruickshanks J, Skeoch CH. Step by step guide: transporting neonates safely. J Neonatal Nurs 2003;1(suppl):9.

23 Auerbach PS, Morris JA Jr, Phillips JB, et al. An analysis of ambulance accidents in Tennessee. JAMA 1987;258:487-90.

24 Barry P, Leslie A, eds. Equipment and monitoring. In: Paediatric and neonatal critical care transport. London: BMJ Books, 2003.

25 Sedin G, Agostino R, Chabernaud J-L, et al. Technical aspects of neonatal transport in Europe. Prenat Neonatal Med 1999;4(suppl 1):35-45.

26 Kinsella JP, Griebel J, Schmidt JM, et al. Use of inhaled nitric oxide during interhospital transport of newborns with hypoxaemic respiratory failure. Pediatrics 2002; 109:158-61.

27 Phelan B. Room ventilation system as a scavenger during inhaled nitric oxide therapy. Proceedings of the 14th conference on high frequency ventilation of infants and children, Snowbird (Utah), 1997.

28 O'Connor T, Greuber R. Transcutaneous measurement of carbon dioxide tension during long-distance transport of neonates receiving mechanical ventilation. J Perinatol 1998;18:189-92.

29 Field D, Milligan D, Skeoch C, et al. Neonatal transport: time to change? Arch Dis Child Fetal Neonatal Ed 1997;76:F1-2. 